\title{
Pancreatic Ductal Adenocarcinoma: Basic and Clinical Challenges for Better Prognosis
}

\section{Toshiyuki Ishiwata*}

\section{Departments of Pathology and Integrative Oncological Pathology, Nippon Medical School, Tokyo, Japan}

Pancreatic cancer, one of the most lethal types of tumors, is the fourth and fifth leading cause of cancer-related death in the USA and Japan, respectively [1]. Pancreatic Ductal Adenocarcinoma (PDAC) - a major histological subtype, comprising $90 \%$ of all pancreatic cancerdisplays local invasion and distant metastasis during early disease stages. This aggressiveness leads to an extremely poor prognosis with an overall survival rate of only $5 \%$ [1]. Surgery offers the only chance for a cure for PDAC; however, $80 \%$ of PDAC patients are considered to be inoperable at diagnosis. No curative treatment is available for advanced stages of the disease. Adjuvant chemotherapies or chemoradiotherapies with 5-FU, gemcitabine, cisplatin, interferon alfa2b, or erlotinib have successfully reduced tumors or prolonged prognosis, but the beneficial effects of these treatments are limited [2-6]

Even after surgery, the five-year survival rates for PDAC remain low, ranging from $15-20 \%$, with most patients dying due to metastatic disease or local recurrence [7]. Thus, the development of early diagnostic methods and new therapeutic approaches for PDAC is a high priority. Serum levels of CA19-9 and CEA are the best known PDAC tumor markers, but they are not useful for early diagnosis. Recently, high levels of microRNAs-including miR-1290 [8], miR-192 [9], miR-99a, miR-155, miR-16, miR-185, miR191, miR-196a, miR-20a, miR-200a, miR-200b, miR-210, miR-21, miR-210, miR-221, miR-24, and miR27a-3p [10] - have been detected in the serum of PDAC patients and these miRNAs are expected to become novel tumor markers for early stages of PDAC.

Improving the prognosis of PDAC will require approaches of both basic and clinical research. For this special issue about PDAC, I have selected two basic research papers and three clinical papers that cover a broad range of topics related to developing new and more effective PDAC treatment methods.

Recent studies have shown that Cancer Stem Cells (CSCs) have self-renewal ability, undergoing multilineage differentiation that is critically important in cancer cell proliferation, invasion, metastasis, and recurrence [11]. Nestin is a class VI intermediate filament protein that is expressed in a variety of stem/progenitor cells, including pancreatic exocrine progenitor cells [12]. Furthermore, nestin is a CSC markers for glioblastoma [13], malignant melanoma [14], and PDAC [15]. Nestin expression is increased in pancreatic intraepithelial lesions according to grade [16], and nestin inhibition decreases PDAC migration and metastasis [17]. These findings suggest that nestin plays important roles in PDAC carcinogenesis and progression, and that it is a candidate therapeutic target for PDAC treatment. Matsuda's review paper focuses on the regulatory mechanisms of nestin, and the correlation between nestin and CSCs in PDAC.

A major cause of the poor prognosis of PDAC is the frequent metastasis of PDAC cells to the liver, lungs, and other organs. Thus, the development of anticancer drugs to inhibit remote metastasis is important for PDAC treatment. Many studies have evaluated tumor proliferation and metastasis using orthotopic implantation of human PDAC cells in immunodeficiency mice. It is possible to monitor tumor size and metastatic status in real time without animal sacrifice using in vivo imaging systems, including ultrasound, positron tomography (PET), CT, MRI, and fluorescent and bioluminescent imaging. However, it is technically difficult to accurately and noninvasively detect intraperitoneal tumors. Dr. Yoshimura established luciferase-transfected PANC-1 cells, and successfully performed in vivo bioluminescence imaging in orthotopic, intravenous injection, and intraperitoneal implantation models of NOG mice with severe immunodeficiency, including $\mathrm{T}$ and $\mathrm{B}$ cell dysfunction, macrophage and complement depression, and defectiveness of natural killer and dendritic cells [18]. This model is expected to be an effective method for analyzing the mechanisms of metastasis and for developing antimetastatic drugs for PDAC.

Even among patients who receive curative resection for PDAC, a high percentage experiences remote metastasis, which can lead to poor prognosis. In search of a novel therapeutic reagent for PDAC, Dr. Kang et al. studied the mushroom species Phellinus linteus (PL). PL and extracts from PL are reportedly effective anti-cancer agents for various malignant tumors, including hepatocellular carcinoma, melanoma, lung cancer, lymphomas, colon cancer, and prostate cancers $[19,20]$. In their review paper, Dr. Kang et al. summarize the underlying mechanisms of the anti-cancer effects of PL and its extracts. Furthermore, they retrospectively investigated the effects of commercially available PL polysaccharides (Aclang) in PDAC patients after surgical resection; adjuvant chemotherapy combined with gemcitabine or 5-FU and Aclang resulted in statistically longer diseasefree survival times compared with in the non-Aclang-treated group. Although this study was based on only a small number of patients, PL and its extracts are expected to be a promising novel adjuvant therapy for PDAC when used in combination with other anti-PDAC drugs.

While adjuvant therapy can improve survival, the effectiveness remains limited. Neoadjuvant therapy has several advantages, including the possibility for early treatment of occult micrometastatic disease such that therapy is delivered to an undisturbed and wellvascularized tumor, as well as the ability to assess drug activity in vivo during surgery [21]. In some cases, neoadjuvant therapy reduces tumor size or metastatic foci, downstaging tumors and thus enabling less radical operation. In resectable pancreatic cancer, neoadjuvant chemotherapy with gemcitabine and cisplatin is associated with a high resection rate and a favorable survival rate [22]. Dr. Matsushita et al.

*Corresponding author: Toshiyuki Ishiwata, Departments of Pathology and Integrative Oncological Pathology, Nippon Medical School, 1-1-5 Sendagi, Bunkyo-ku, Tokyo, 113-8602 Japan, Tel: +81-3-3822-2131 ext. (5232); Fax: +81-35814-6274; E-mail: ishiwata@nms.ac.jp

Received June 19, 2013; Accepted June 27, 2013; Published July 10, 2013

Citation: Ishiwata T (2013) Pancreatic Ductal Adenocarcinoma: Basic and Clinical Challenges for Better Prognosis. J Carcinogene Mutagene S9: 005. doi:10.4172/2157-2518.S9-005

Copyright: @ 2013 Ishiwata T. This is an open-access article distributed under the terms of the Creative Commons Attribution License, which permits unrestricted use, distribution, and reproduction in any medium, provided the original author and source are credited. 
Citation: Ishiwata T (2013) Pancreatic Ductal Adenocarcinoma: Basic and Clinical Challenges for Better Prognosis. J Carcinogene Mutagene S9: 005. doi:10.4172/2157-2518.S9-005

Page 2 of 2

administered neoadjuvant chemotherapy with gemcitabine and S-1 (NeoGS) in resectable and borderline resectable PDAC patients. This study included only a small number of patients, but NeoGS therapy showed favorable results with a $27 \%$ partial response, and high R0 resection and low lymph node metastasis rates.

In resectable PDAC patients, laparoscopic pancreatectomy is one applicable technique. Laparoscopic surgery has the benefit of improved visualization, but also the disadvantage of a limited sense of touch. Laparoscopic surgery is less invasive, making it particularly useful in PDAC patients of advanced age or with complicated diseases. Dr. Nakamura and his colleagues have performed laparoscopic pancreatectomies in 148 patients, including 25 PDAC cases [23,24]. In this review, the authors describe their laparoscopic surgery techniques for PDAC, and focus on the oncologic outcomes and long-term outcomes of laparoscopic surgery for PDAC patients.

Overall, this special issue covers a broad range of PDAC-related topics, including CSC markers for PDAC, a newly established metastatic mouse model, an anti-PDAC reagent from a mushroom species, novel neoadjuvant chemotherapy, and laparoscopic pancreatectomy. The new information from each of these avenues of investigation greatly contributes to the progress of basic and clinical research towards improving the treatment and prognosis of patients with PDAC.

\section{References}

1. Siegel R, Ward E, Brawley O, Jemal A (2011) Cancer statistics, 2011: the impact of eliminating socioeconomic and racial disparities on premature cancer deaths. CA Cancer J Clin 61: 212-236.

2. Neoptolemos JP, Stocken DD, Friess H, Bassi C, Dunn JA, et al. (2004) A randomized trial of chemoradiotherapy and chemotherapy after resection of pancreatic cancer. N Engl J Med 350: 1200-1210.

3. Oettle H, Post S, Neuhaus P, Gellert K, Langrehr J, et al. (2007) Adjuvant chemotherapy with gemcitabine vs observation in patients undergoing curativeintent resection of pancreatic cancer: a randomized controlled trial. JAMA 297 : 267-277.

4. Neoptolemos JP, Stocken DD, Bassi C, Ghaneh P, Cunningham D, et al. (2010) Adjuvant chemotherapy with fluorouracil plus folinic acid vs gemcitabine following pancreatic cancer resection: a randomized controlled trial. JAMA 304 : 1073-1081.

5. Regine WF, Winter KA, Abrams RA, Safran H, Hoffman JP, et al. (2008) Fluorouracil vs gemcitabine chemotherapy before and after fluorouracilbased chemoradiation following resection of pancreatic adenocarcinoma: a randomized controlled trial. JAMA 299: 1019-1026.

6. Picozzi VJ, Abrams RA, Decker PA, Traverso W, O'Reilly EM, et al. (2011) Multicenter phase II trial of adjuvant therapy for resected pancreatic cance using cisplatin, 5-fluorouracil, and interferon-alfa-2b-based chemoradiation: ACOSOG Trial Z05031. Ann Oncol 22: 348-354.

7. Ahrendt SA, Pitt HA (2002) Surgical management of pancreatic cancer. Oncology (Williston Park) 16: 725-734.

8. Li A, Yu J, Kim H, Wolfgang CL, Canto MI, et al. (2013) MicroRNA Array Analysis Finds Elevated Serum miR-1290 Accurately Distinguishes Patients with Low-Stage Pancreatic Cancer from Healthy and Disease Controls. Clin Cancer Res 19: 3600-3610.

9. Zhao C, Zhang J, Zhang S, Yu D, Chen Y, et al. (2013) Diagnostic and biological significance of microRNA-192 in pancreatic ductal adenocarcinoma. Oncol Rep 30: 276-284.

10. Humeau M, Torrisani $J$, Cordelier $P$ (2013) miRNA in clinical practice: Pancreatic cancer. Clin Biochem 46: 933-936.

11. Li Y, Laterra J (2012) Cancer stem cells: distinct entities or dynamically regulated phenotypes? Cancer Res 72: 576-580.

12. Esni F, Stoffers DA, Takeuchi T, Leach SD (2004) Origin of exocrine pancreatic cells from nestin-positive precursors in developing mouse pancreas. Mech Dev 121: 15-25.

13. Lu WJ, Lan F, He Q, Lee A, Tang CZ, et al. (2011) Inducible expression of stem cell associated intermediate filament nestin reveals an important role in glioblastoma carcinogenesis. Int J Cancer 128: 343-351.

14. Akiyama M, Matsuda Y, Ishiwata T, Naito Z, Kawana S (2013) Inhibition of the stem cell marker nestin reduces tumor growth and invasion of malignant melanoma. J Invest Dermatol 133: 1384-1387.

15. Matsuda $Y$, Kure S, Ishiwata T (2012) Nestin and other putative cancer stem cell markers in pancreatic cancer. Med Mol Morphol 45: 59-65.

16. Kure S, Matsuda Y, Hagio M, Ueda J, Naito Z, et al. (2012) Expression of cancer stem cell markers in pancreatic intraepithelial neoplasias and pancreatic ductal adenocarcinomas. Int J Oncol 41: 1314-1324.

17. Matsuda Y, Naito Z, Kawahara K, Nakazawa N, Korc M, et al. (2011) Nestin is a novel target for suppressing pancreatic cancer cell migration, invasion and metastasis. Cancer Biol Ther 11: 512-523.

18. Suemizu H, Monnai M, Ohnishi $Y$, Ito M, Tamaoki N, et al. (2007) Identification of a key molecular regulator of liver metastasis in human pancreatic carcinoma using a novel quantitative model of metastasis in NOD/SCID/gammacnull (NOG) mice. Int J Oncol 31: 741-751.

19. Kojima H, Tanigawa N, Kariya S, Komemushi A, Shomura Y, et al. (2006) A case of spontaneous regression of hepatocellular carcinoma with multiple lung metastases. Radiat Med 24: 139-142.

20. Nam SW, Han JY, Kim JI, Park SH, Cho SH, et al. (2005) Spontaneous regression of a large hepatocellular carcinoma with skull metastasis. J Gastroenterol Hepatol 20: 488-492.

21. Paulson AS, Tran Cao HS, Tempero MA, Lowy AM (2013) Therapeutic advances in pancreatic cancer. Gastroenterology 144: 1316-1326.

22. Palmer DH, Stocken DD, Hewitt $H$, Markham CE, Hassan AB, et al. (2007) A randomized phase 2 trial of neoadjuvant chemotherapy in resectable pancreatic cancer: gemcitabine alone versus gemcitabine combined with cisplatin. Ann Surg Oncol 14: 2088-2096.

23. Nakamura Y, Uchida E, Nomura T, Aimoto T, Matsumoto S, et al. (2009) Laparoscopic pancreatic resection: some benefits of evolving surgical techniques. J Hepatobiliary Pancreat Surg 16: 741-748.

24. Nakamura Y, Uchida E, Aimoto T, Matsumoto S, Yoshida H, et al. (2009) Clinical outcome of laparoscopic distal pancreatectomy. J Hepatobiliary Pancreat Surg 16: 35-41.

\footnotetext{
This article was originally published in a special issue, Pancreatic Ductal Adenocarcinoma handled by Editor(s). Dr. Toshiyuki Ishiwata, Nippon Medical School, Japan
} 